\title{
Una regla de predicción fue útil para identificar pacientes con síncope con mayor riesgo a corto plazo
}

\section{Objetivo}

Aunque las causas de síncope son usualmente benignas, se asocian ocasionalmente con una importante morbimortalidad. El presente estudio quiere desarrollar una regla de predicción que pueda identificar pacientes con riesgo aumentado a corto plazo y guiar decisiones de internación.

\section{Diseño}

Estudio de cohortes prospectivo*

\section{Lugar}

Guardia de Hospital Universitario, San Franciso, EE.UU

\section{Pacientes}

Sujetos que se presentaban con síncope o pre-síncope.

\section{Evaluación de Factores Pronósticos}

Los médicos llenaban en forma prospectiva un cuestionario estandarizado que evaluaba posibles factores pronósticos (50 variables de la historia clínica, el examen físico, tests y hallazgos de laboratorio). Luego de un análisis univariado entre los factores pronósticos y los resultados, se seleccionó a aquellos asociados con una $p<0.1$ y una aceptable concordancia interobservador (índice kappa $>0.5$ ) $^{*}$ para incluir en el análisis multivariado* $y$ derivar la regla de predicción.

\section{Medición de Resultados Principales}

El resultado principal que intentó predecir la regla de predicción fueron eventos adversos serios a los 7 días de la visita a la guardia. Se definió antes del estudio a los eventos adversos como muerte, infarto de miocardio, arritmia, tromboembolismo pulmonar, accidente cerebrovascular, hemorragia subaracnoidea, hemorragia significativa, o cualquier condición que cause una nueva visita o internación por un evento relacionado.

\section{Resultados Principales}

De los 684 pacientes que realizaron visitas por síncope, 79 (11.5\%) de las mismas resultaron en pacientes con eventos serios $(8.2 \%$ causas cardíacas). La regla de decisión final quedó conformada por las siguientes variables: 1) Electrocardiograma (ECG) anormal (definido como ausencia de ritmo sinusal o nuevos cambios en relacion a ECG previos); 2) Disnea; 3) Hematocrito $<30 \%$; 4) Tensión arterial sistólica inicial $<90 \mathrm{mmHg}$; 5) Historia de insuficiencia cardíaca congestiva. Si cualquiera de las variables previas es afirmativa, se considera como un resultado positivo de la regla. Si se aplicara a este grupo, la aplicación de la regla podría haber reducido las admisiones un $10 \%$.

Tabla. Performance diagnóstica de la Regla de Síncope de San Francisco

\begin{tabular}{c|c|c|c|c} 
Resultado & $\begin{array}{c}\text { Sensibilidad* } \\
\text { (IC 95\%) }\end{array}$ & $\begin{array}{c}\text { Especificidad** } \\
\text { (IC 95\%) }\end{array}$ & $\begin{array}{c}\text { Coeficiente de } \\
\text { Probabilidad } \\
\text { Positiv0* } \\
\text { (IC 95\%) }\end{array}$ & $\begin{array}{c}\text { Coeficiente de } \\
\text { Probabilidad } \\
\text { Negativo* } \\
\text { (IC 95\%) }\end{array}$ \\
\hline $\begin{array}{c}\text { Alguna de los } \\
\text { cinco factores de } \\
\text { riesgo presente }\end{array}$ & $\begin{array}{c}96,2 \% \\
(92 \text { a } 100 \%)\end{array}$ & $\begin{array}{c}61,9 \% \\
(58 \text { a66\%) }\end{array}$ & $\begin{array}{c}2,53 \\
(2,3 \text { a } 2,8)\end{array}$ & $\begin{array}{c}0,06 \\
(0,02 \text { a } 0,19)\end{array}$ \\
\hline
\end{tabular}

\section{Conclusiones}

La Regla de Síncope de San Franciso derivada en esta cohorte parece ser sensible para detectar pacientes con riesgo a corto plazo de eventos serios. De ser validada prospectivamente, podría ser utilizada para ayudar en la toma de decisiones en pacientes que consultan por síncope.

Fuente de financiamiento: sin financiamiento externo.

\section{Comentario}

El síncope es un motivo de consulta frecuente, una de cada cuatro personas lo padecen alguna vez, y se estima que motiva el 1 al $2 \%$ de las consultas a la guardia o internaciones hospitalarias. Las posibles etiologías son muchas, y van desde las de buen pronóstico como el desmayo común (también llamado vasovagal o neurocardiogénico) hasta las potencialmente fatales como las arritmias severas ${ }^{1}$

La decisión de internación en pacientes con síncope es muchas veces arbitraria y basada en recomendaciones de expertos, y algunos estudios muestran que incluso en centros académicos, se decide internar pacientes que terminan teniendo causas benignas ${ }^{2}$. En este sentido, es muy interesante poder contar con una regla para permitir identificar a los pacientes de bajo riesgo, y así poder manejarlos ambulatoriamente. Este estudio intenta resolver esta interesante pregunta, y sugiere que la ausencia de cinco factores de riesgo, fáciles de evaluar en una guardia de baja complejidad, podría servir a tales fines. Como uno sobretodo no quiere dejar de internar a alguien de alto riesgo, la sensibilidad* es la característica operativa que se debe maximizar:así, si la regla me da un resultado negativo, prácticamente descartaría la posibilidad de que el paciente tenga un evento serio en el corto plazo, por lo que se podría tomar una conducta ambulatoria.

La principal limitación del presente estudio, como lo afirman sus autores, es la falta de la validación de esta regla, necesaria para recomendar su aplicación en la práctica cotidiana. Esta validación sirve para contestar la siguiente pregunta: ¿la regla que funcionó en este grupo particular de pacientes en la que se derivó la regla; sirve también cuando se aplica a un grupo distinto de pacientes que consultan por síncope ${ }^{3}$ En lo que respecta a otras preguntas de interés al médico que ejerce medicina ambulatoria, sería interesante evaluar el funcionamiento de esta regla en ámbitos distintos a una guardia como el consultorio o el domicilio del paciente (aunque es necesario un ECG para implementarla).

\section{Conclusiones del comentador}

Aunque esta simple regla parece prometedora para descartar pacientes de mayor riesgo, aun se requieren estudios confirmatorios que la validen para recomendar su utilización en la guardia, así como en otros posibles ámbitos.

Dr. Federico Augustovski [ Unidad de Medicina Familiar y Preventiva Hospital Italiano de Buenos Aires ]

Augustovski F. Una regla de predicción fue útil para identificar pacientes con síncope con mayor riesgo a corto plazo. Evid. actual. práct. ambul 2004;7:109. Comentado de: Quinn JV, Stiell IG, McDermott DA, Sellers KL, Kohn MA, Wells GA. Derivation of the San Francisco Syncope Rule to predict patients with short-term serious outcomes. Ann Emerg Med. 2004 Feb;43(2):224-32.

\section{Referencias}

Síncope. Publicado en "Medicina Familiar y Práctica Ambulatoria". Augustovski F. Capítulo 81. Editores Rubinstein A, Terrasa S, Rubinstein E, Durante E, Carrete P. Editorial Médica Panamericana, Marzo de 2001. pp 587-96

2.Quinn JV; Mc DermottDA; Stiell IG., et al. Physician judgement in evaluating patients with syncope. Acad Emerg Med. 2002;9:452-53

3.McGinn TG, Guyatt GH, Wyer PC, Naylor CD, Stiell IG, Richardson WS. Users' guides to the medical literature: XXII: how to use articles about clinical decision rules. Evidence-Based Medicine Working Group. JAMA. 2000 Jul 5;284(1):79-84. 